\title{
Local density determines nuclear movements during syncytial blastoderm formation in a cricket
}

\author{
Seth Donoughe ${ }^{1,2, *}$, Jordan Hoffmann ${ }^{3}$, Taro Nakamura ${ }^{1,4}$, Chris H. Rycroft ${ }^{3,5, *}$, Cassandra G. Extavour ${ }^{1,6, *}$
}

\begin{abstract}
Animal embryos pass through an early stage called the blastoderm, in which cells are arranged in a continuous layer at the periphery of the embryo. Despite the broad evolutionary conservation of this embryonic stage, the cellular behaviours that lead to blastoderm formation vary across animals, and the mechanisms that regulate these behaviours are poorly understood. In most insects, pre-blastoderm development begins as a syncytium: that is, many nuclei divide and move throughout the single shared cytoplasm of the embryo. Then these syncytial nuclei must move from their scattered positions within the cytoplasm to form a single layer at the cortex. Recent work showed that in the fruit fly Drosophila melanogaster, some of these early nuclear movements are caused by pulses of cytoplasmic flows that are coupled to synchronous divisions. Here, we show that the cricket Gryllus bimaculatus has an altogether different solution to the problem. We quantified nuclear dynamics during the period of syncytial cleavages and movements that lead to blastoderm formation in G. bimaculatus embryos with transgenically labeled nuclei. We found that: (1) cytoplasmic flows were unimportant for nuclear movement, and (2) division cycles, nuclear speeds, and the directions of nuclear movement were not synchronized across the embryo as in D. melanogaster, but instead were heterogeneous in space and time. Moreover, several aspects of nuclear divisions and movements were correlated with local nuclear density. We show that previously proposed models for the movement of $D$. melanogaster syncytial nuclei cannot explain the behaviours of $G$. bimaculatus syncytial nuclei. We introduce a novel geometric model based on asymmetric local pulling forces on nuclei, which recapitulates the density-dependent nuclear speeds and orientations of unperturbed $G$. bimaculatus embryos, without invoking the common paradigms of localized polarity cues or cell lineage as determinants of nuclear activity. Our model also accurately predicts nuclear behavior in embryos physically manipulated to contain regions of atypical nuclear densities. We show that this model can be used to generate falsifiable predictions about the dynamics of blastoderm formation in other insect species.
\end{abstract}

\section{Introduction}

Proper positioning of nuclei is essential for cellular function ${ }^{1,2}$. The task of correctly positioning nuclei is further specialized in syncytial cells - those with multiple nuclei sharing the same cytoplasm ${ }^{3-5}$. Naturally occurring syncytia include animal muscle cells, heterokaryotic fungi, plant endosperm, and early cleavage stage arthropod embryos $^{6-9}$. Among arthropods, there have likely been multiple independent evolutionary origins of a syncytial phase of embryonic development ${ }^{7}$. Here, we focus on insects.

When an insect egg is fertilized, the oocyte and sperm pronuclei fuse, forming the zygotic nucleus within a single, large

\footnotetext{
${ }^{*}$ Corresponding authors. E-mail: donoughe@uchicago .edu, chr@seas .harvard. edu, extavour@oeb.harvard.edu

${ }^{1}$ Department of Organismic and Evolutionary Biology, Harvard University, Cambridge, MA, USA

${ }^{2}$ Present address: Department of Molecular Genetics and Cell Biology, University of Chicago, Chicago, IL, USA

${ }^{3}$ John A. Paulson School of Engineering and Applied Sciences, Harvard University, Cambridge, MA, USA

${ }^{4}$ Present address: Division of Evolutionary Development, National Institute for Basic Biology, Okazaki, Japan

${ }^{5}$ Computational Research Division, Lawrence Berkeley Laboratory, Berkeley, CA, USA

${ }^{6}$ Department of Molecular and Cellular Biology, Harvard University, Cambridge, MA, USA
} 
cell $^{10,11}$. In most insect taxa there follows a series of syncytial cleavages-nuclear divisions without cytokinesis ${ }^{10-12}$. As the divisions proceed, nuclei move throughout the cytoplasm of the embryo. Although some nuclei remain submerged in the middle of the embryo, most of them travel into the periplasm, a region of cytoplasm at the periphery of the embryo ${ }^{10,11}$. The nuclei in the periplasm comprise a syncytial blastoderm, a single layer of nuclei surrounding the cytoplasm in the interior ${ }^{10,11}$.

Syncytial blastoderm formation has been studied most closely in the fruit fly Drosophila melanogaster ${ }^{13,14}$. After fertilization, D. melanogaster undergoes 13 synchronous divisions ${ }^{13}$. During cycles 4 through 6 , nuclei spread out along the anterior-posterior (A-P) axis without entering the periplasm (a process referred to as "axial expansion") ${ }^{14-17}$. Nuclear movements along the A-P axis appear to be caused by the contraction of a subset of the cortex, which generates a cytoplasmic flow that carries nuclei towards the poles ${ }^{18,19}$. It has also been suggested that local forces act on nuclei via their astral microtubules (MTs) pulling the nuclei toward the adjacent F-actin network ${ }^{20}$ and/or mutual repulsion among neighboring nuclei ${ }^{21}$. Then, during cycles 7 through 9 , the nuclei simultaneously move into the periplasm (leaving a small subset behind as "yolk nuclei," also called "vitellophages") ${ }^{11,21,22}$. Finally, during cycles 10 through 13 , the nuclei remain in the periplasm, arranged as a single layer ${ }^{16,22,23}$. They increase in local nuclear density and take on orderly geometric spacing ${ }^{24-29}$.

Among insects with syncytially cleaving embryos, it appears to be universal that nuclei travel through the interior cytoplasm and into the periplasm ${ }^{10,11,30}$, yet species differ dramatically with respect to the timing, speeds, and the paths that their nuclei traverse while getting there ${ }^{10,11,30}$. This raises the question of how different insect embryos generate such embryological diversity. There is evidence from fixed preparations that some of the mechanisms described in D. melanogaster — namely cytoplasmic flows and MT-mediated pulling-might be operating in more distantly related insects ${ }^{31,32}$. To assess such possibilities, quantitative, nucleus-level data on the dynamics of syncytial blastoderm formation are needed for species other than D. melanogaster. Therefore, we set out to investigate an informative comparator: the two-spotted field cricket Gryllus bimaculatus (order: Orthoptera).

G. bimaculatus is a powerful complement to D. melanogaster for the study of syncytial development. G. bimaculatus embryos are larger (approximately five-fold longer and three-fold wider) ${ }^{33,34}$ and their blastoderm formation occurs more slowly $\left(14 \text { hours at } 28.5^{\circ} \mathrm{C} \text { versus } 3 \text { hours at } 25^{\circ} \mathrm{C}\right)^{13,35}$. G. bimaculatus is hemimetabolous, and its embryonic development differs in many respects from that of $D$. melanogaster and other model holometabolous insect species ${ }^{34}$. It likely retains many features of ancestral insect embryogenesis, unlike the relatively derived fruit fly model ${ }^{34}$. Crucially, a transgenic line of $G$. bimaculatus expressing a constitutive ubiquitous Histone2B-Enhanced Green Fluorescent Protein fusion has been generated ${ }^{35}$. The fusion protein presents a strong fluorescent contrast between syncytial nuclei and the surrounding cytoplasm during the entirety of pre-blastoderm development ${ }^{35}$. This enabled us to record, track, and analyze the movements of nuclei during syncytial development, starting from mitotic cycle $\sim 2-4$ and ending at the formation of the blastoderm.

We used multiview lightsheet and confocal microscopy to capture three-dimensional timelapse (3D+T) datasets and epifluorescence microscopy to capture two-dimensional timelapse $(2 \mathrm{D}+\mathrm{T})$ datasets of syncytial development. We used a semi-automated approach to reconstruct nuclear tracks through space ${ }^{36,37}$, and analyzed nuclear divisions, speeds, and movement orientations. We show that each of these nuclear behaviours co-varies predictably with local nuclear density rather than with axial position, lineage, or developmental timing. We also show that the patterns of nuclear migration are more consistent with active movement through the cytoplasm, rather than with passive movement resulting from being carried along in a cytoplasmic flow. Based on our empirical description, and inspired by previously published work on active nuclear migration in other contexts ${ }^{20,38-41}$, we built a simple computational model of nuclear movement based on asymmetric pulling forces and local interactions among nuclei. This model recapitulates the main features of G. bimaculatus nuclear divisions, speeds, and orientations during syncytial development. We test the model by experimentally altering nuclear density, and find support for the hypothesis that changes to nuclear density cause changes to nuclear movement. Finally, we use this model to generate falsifiable hypotheses about blastoderm formation in other insect species. 


\section{Methods}

Transgenesis and animal culture: G. bimaculatus cultures were maintained as previously described ${ }^{34}$. We used an established nucleus-marked transgenic line of $G$. bimaculatus ${ }^{35}$, in which the endogenous actin promoter drives expression of the G. bimaculatus Histone 2B (H2B) protein fused to Enhanced Green Fluorescent Protein (EGFP) (this transgenic line is abbreviated hereafter as Act-H2B-EGFP). To label cytoplasm and nuclei together, we generated a new transgenic insertion of a myristoylated and palmitoylated tandem dimer Tomato fluorescent protein (hereafter: $\operatorname{mtd} \mathrm{T}$ ) expressed under the control of the G. bimaculatus actin promoter. The mtdT-3xHA sequence was obtained from pUASTattB-mtdT-3XHA ${ }^{42}$ (Addgene Plasmid \#24355), and cloned into the pXL[Gbact-GFP-pA] vector ${ }^{35}$ in place of GFP, to create $\mathrm{pXL}$ [Gbact-mtdT-3xHA-pA]. We used this plasmid to generate a stable transgenic line of G. bimaculatus following previously described methods ${ }^{35}$. We crossed the Act-mtdT and Act-H2B-EGFP lines to obtain mature F1 females with both transgenes, assessed by using a fluorescence dissection microscope to check for red and green fluorescent protein expression in late embryogenesis. These F1 females with both transgenes matured to adulthood, and then were crossed to wild-type males. F2 eggs were collected for live imaging. Thus, when imaging the mtdT and H2B-EGFP transgenic proteins in the same embryo, both were maternally provided to the embryos.

Collecting and culturing embryos: To collect embryos for live imaging, females were allowed to lay eggs in damp sand for two hours at a time, following previously described methods ${ }^{34}$. Embryos were examined on a fluorescence dissection microscope within five hours of collection. If there were between 2 and 8 nuclei visible, then embryos were mounted for microscopy as described below under Microscopy. After imaging, embryos were placed in a 10 mm diameter plastic petri dish (VWR 25384-342), the bottom of which had been covered with Kimwipes (VWR 21905-026) moistened with distilled water. We incubated the dish at $28.5^{\circ} \mathrm{C}$ so that embryos could continue to develop. We checked embryos daily and removed any dead ones. Only datasets from embryos that hatched within 16 days of being laid were used for analysis.

Microscopy: For 3D+T lightsheet imaging, we mounted embryos individually in a column of $1 \%(\mathrm{w} / \mathrm{v})$ low-melt agarose (Bio-Rad 1613112) in distilled water. Suspended in the mounting agarose were $1-\mu \mathrm{m}$ diameter red fluorescent polystyrene beads (ThermoFisher F8821) at $0.015 \%$ of the stock concentration. Lightsheet imaging was conducted with a Zeiss Z.1 Lightsheet microscope controlled by Zen Black software (Zeiss), with the agarose column immersed in a bath of distilled water, temperature controlled at $28.5^{\circ} \mathrm{C}$. Embryos were imaged one at a time, positioned with the A-P axis oriented vertically. For each time point, $z$-stacks were captured at $72^{\circ}$ or $90^{\circ}$ increments, rotationally distributed about the long axis of the egg. Data were simultaneously captured with $488 \mathrm{~nm}$ and $568 \mathrm{~nm}$ lasers at a time interval of 90 seconds, with 100 to 200 optical sections per $z$-stack. Among lightsheet datasets, $z$-step size ranged from 4 to $10 \mu \mathrm{m}$, depending on the overall size of the field of view needed to capture the embryo. For 3D+T imaging of cytoplasm and nuclei together, we individually mounted embryos in a glass-bottom dish (MatTek P06G-1.5-20-F) in a $20 \mu \mathrm{L}$ puddle of molten $0.5 \%(\mathrm{w} / \mathrm{v})$ low-melt agarose in distilled water. Then we covered the immobilized embryos in distilled water and imaged them on a Zeiss LSM 880 confocal microscope at $28.5^{\circ} \mathrm{C}$. For $2 \mathrm{D}+\mathrm{T}$ imaging of whole embryos, they were mounted in agarose microwells as previously described ${ }^{43}$ and imaged using epifluorescence on a Zeiss Cell Discoverer microscope with a 5x objective, controlled with Zen Blue (Zeiss). Epifluorescence datasets were captured as a $z$-stack at each time point. Embryo constrictions were conducted with a custom device that is described in the Supplemental Information. A design file of the device's components is included as Supplemental File 3.

Image processing and segmentation: Lightsheet datasets were processed using the Multiview Reconstruction plug-in for Fiji ${ }^{44,45}$. In epifluorescence datasets, $z$-slices were combined using the Extended Depth of Focus function (Contrast mode) in Zen Blue (Zeiss). Confocal datasets were processed in Fiji to generate maximum intensity projections. Nucleus tracks were generated with Ilastik ${ }^{37}$ and manually corrected with Fiji plug-in MaMuT ${ }^{36}$. Additional image processing details are included in the Supplemental Information.

Measuring and simulating quantitative features of nuclear behaviour: Data analysis and simulations of nuclear 
movements were performed using custom scripts written in Mathematica (Wolfram) and Python. We calculated nuclear speed, local nuclear density, rate of change in number of nuclei, and movement toward nearby unoccupied space. See Supplemental Information for the specifics of these calculations and a detailed description of nuclear movement simulations. Code to simulate nuclear movements is available at the GitHub repository for this project: https://github.com/hoffmannjordan/gryllus_nuclear_movements.

Preparing figures: Micrographs for presentation were processed in Fiji ${ }^{46}$. Figures were generated with Mathematica and assembled with Illustrator (Adobe).

\section{Results and Discussion}

The G. bimaculatus syncytial blastoderm forms during approximately eight hours of development at $28.5^{\circ} \mathrm{C}$, followed by cellularization and coalescence of the embryonic rudiment ${ }^{34,35}$ (Fig. 1A). We recorded G. bimaculatus syncytial blastoderm formation in toto by using 3D+T lightsheet microscopy to image H2B-EGFP transgenic embryos. We tracked the movements and divisions of nuclei as they expanded throughout the embryo, which enabled us to reconstruct nuclear lineages (Fig. 1B) and quantify nuclear density, speed, and direction of movement. Similar to $D$. melanogaster ${ }^{14,16,17}$, some nuclei appear to move in a highly directed manner toward the poles of the embryo (Fig. 1B, arrowhead). Unlike D. melanogaster, however, G. bimaculatus nuclei move into the periplasm asynchronously, with some reaching the periplasm 240 minutes after those that first reached it (Fig. 1B).

\section{Nuclei are not predominantly moved by cytoplasmic flows}

We wanted to determine whether nuclei are moved passively by being carried along in a cytoplasmic flow ${ }^{18,19}$, or instead moved by a local active force $e^{20,21,32,39}$. We generated a new stable transgenic line of G. bimaculatus that expressed myristoylated and palmitolyated mTomato $(\mathrm{mtdT})$ protein in the embryo. The mtdT protein is predominantly localized to the cytoplasm in the immediate vicinity of each syncytial nucleus (i.e. within the "energid"7,10). It was excluded from putative yolk granules (Fig. 1C, white arrowheads; SI File 2), but allowed us to see their outlines and follow their movements. We generated embryos that co-expressed mtdT and H2B-EGFP, which enabled us to image yolk granules and nuclear movements together (Fig. 1C; SI File 2). We found that yolk granules and adjacent nuclei do not tend to move in the same direction together, even when they are quite close in space (e.g. within $40 \mu \mathrm{m}$, as shown in Fig. 1C and SI File 2). This is inconsistent with a cytoplasmic flow that moves yolk granules and nuclei together. To further test the possibility of movement via cytoplasmic flows, we computed instantaneous pairwise correlations between the movement vectors of all pairs of nuclei. If nuclei were embedded in a cytoplasmic flow, we would expect nearby nuclei to move more similarly to one another than nuclei that were farther apart. This was not the case. Irrespective of pairwise distance, pairs of nuclei exhibit a random pattern of movement correlations (overall correlation of pairwise values with separation distance yields $R^{2}=0.032$; Fig. 1D; this calculation, and those presented in Fig. 2 and Fig. 3, was done on a dataset with 310,741 nucleus-timepoints). These observations suggest that $G$. bimaculatus syncytial nuclei are not predominantly moved by cytoplasmic flows.

\section{Mitotic cycle duration is positively associated with local nuclear density}

Previous work showed that syncytial nucleus divisions in D. melanogaster are synchronous, and that divisions are coupled to the nuclear movements that underlie blastoderm formation ${ }^{18,19}$. We asked whether a similar mechanism operates in G. bimaculatus embryos. For a coarse-grained measurement of proliferation, we calculated the percent change in the number of detectable nuclei over time (Fig. 2A). Plotted over time, this metric displays a dynamic series of peaks and valleys, with each peak representing a pulse of divisions. These peaks occur initially at mean intervals of 49 minutes, and the time between peaks increases as development proceeds. This indicates that the cell cycle duration is approximately 49 minutes, which is a much greater time interval than the 8 to 9 minute cell cycle duration in a $D$. melanogaster embryo ${ }^{13}$. The sharpness of the peaks attenuates over time, indicating a decrease in relative 
synchronicity (Fig. 2A). Next, we sought to determine whether the mitotic cycles are collectively going out of phase and/or the mitotic cycle lengths are themselves changing. We measured the cycle length of the nuclei, finding that it changes markedly over the course of blastoderm formation. An example lineage is shown in Fig. 2B. During four successive cycles, mean cycle length increases from 49 to 87 minutes (Fig. 2B). There is also considerable variation in cycle length among nuclei at the same cycle number (e.g. at cycle 4, SD of cycle length $=11$ minutes; Fig. 2B). This is again in contrast to $D$. melanogaster embryos, in which cycle length increases over time, but all nuclei within a cycle have the same period ${ }^{22,47}$. We hypothesized that in $G$. bimaculatus, variation in local nuclear density gives rise to the heterogeneity of cycle length. To test this, we computed the local nuclear density and proliferation time in the vicinity of each nucleus throughout blastoderm formation (three example time points are shown in Fig. 2C-E). Within each time point, we took all possible nucleus pairs and computed the percentage of instances where the nucleus with the higher local density also had a high proliferation time. These percentages are: $t=300 \mathrm{~min}: 91 \%$, $t=353$ min: $88 \%, t=413$ min: $75 \%$. We concluded that there is a positive association between local nuclear density and mitotic cycle length across a range of local densities and throughout syncytial blastoderm formation.

\section{Nuclear speed is biphasic and negatively associated with density}

Given that a single variable-density - helped to explain both spatial and temporal variation in mitotic cycle length, we wondered whether the speeds of nuclear movements also followed a similar coherent pattern. We calculated instantaneous speed of all nuclei and then plotted those speeds over time $(y)$ vs. position along the A-P axis $(x)$ (Fig. 3A). Nuclear speed oscillates back and forth between "fast" and "slow" ( $>4$ to $<1 \mu \mathrm{m}$ per minute, respectively). It appeared that in the central region of the embryo, farthest from the anterior and posterior poles, peak speeds decrease earlier and the speed oscillations dissipate sooner than at either pole (Fig. 3A, middle of A-P axis, $t>150 \mathrm{~min}$ ). To illustrate this further, we ordered the nuclei according to their position along the A-P axis and then partitioned them into three terciles, with equal numbers of nuclei in each one (labeled "anterior", "middle", and "posterior" terciles; Fig. 3B). When we plotted speed over time for the nuclei contained in each tercile, we observed that nuclear speed oscillates for all three terciles. In the middle tercile, however, where density is higher than in the other terciles at every developmental time point examined, the oscillation is less pronounced and diminishes sooner, than in the anterior and posterior terciles (Fig. 3C). We also noticed that the speed oscillations are qualitatively similar to the oscillations of the percent change in total number of nuclei (compare Fig. 2A and 3C). Therefore we hypothesized that each nucleus's movements jointly depend on its local density and on the amount of time spent executing the cell cycle.

To test this, we computed time-since-last-division for each nucleus within the 200 minutes of development depicted in Fig. 3A. We divided the nuclei into three subsets according to their local nuclear density: "low," "medium," and "high" ( $<11, \geq 11$ and $<29, \geq 29$ density units, defined here as the number of nuclei within a $150 \mu \mathrm{m}$ radius). We plotted instantaneous nuclear speed for each density bin, with time re-zeroed to begin at the most recent division of each nucleus. This revealed density-dependent speed oscillations for all nuclei, regardless of chronological age or spatial location within the egg (Fig. 3D). Nuclear speed alternated between relatively fast and slow phases, which we refer to as Phase $\mathrm{A}$ and Phase $\mathrm{B}$, respectively. During Phase $\mathrm{A}$, which is immediately after a division, each daughter nucleus moves relatively quickly (median Phase A speed: $2.3 \mu \mathrm{m}$ per minute) for between 20 and 28 minutes. During Phase B, the nucleus remains largely stationary (median Phase B speed: $0.4 \mu \mathrm{m}$ per minute) for between 10 and 20 minutes before again dividing and repeating this process (Fig. 3D). We plotted nuclear speed ( $y$ ) vs. nuclear density $(x)$ for Phase A and Phase B (Fig. 3E, F), and considering that both speed and density are by definition positive, in each case we fitted an exponential curve with the form $y=y_{0} e^{-x / x_{0}}$. This yields a "density scale" $\left(x_{0}\right)$ that captures how large of a change in density produces a given change in speed. The density scale of Phase $\mathrm{A}$ is 30.4 density units (90\% CI: 28.8 to 32.1). For Phase B it is 120.4 density units (90\% CI: 73.4 to 167.4). In sum, we found that Phase A nuclear movements have overall higher speeds that are strongly associated with density, while Phase B nuclear movements have lower speeds that are weakly associated with local density. We concluded that nuclear speeds covaried with local nuclear density and time in the cell cycle. 


\section{In the cytoplasm, nuclei tend to move into nearby unoccupied space}

Nuclear speed alone cannot achieve axial expansion: nuclei also need to move in appropriate directions to ensure even distribution of nuclei in the blastoderm. The directionality cannot be uniform for all nuclei, however, otherwise all nuclei would end up crowded together in a single region of the egg. To investigate what might predict directionality, we began by observing the global features of nuclear paths during syncytial blastoderm formation. Once syncytial nuclei reach the periplasm, they remain there until cellularization occurs ${ }^{14,35}$ (Fig. 1A, B), suggesting that $G$. bimaculatus nuclei, like $D$. melanogaster nuclei, ultimately become anchored in the periplasm ${ }^{16,48-51}$. Compared to the synchronously emerging nuclei of $D$. melanogaster ${ }^{16}$, however, $G$. bimaculatus nuclei move along trajectories that are varied, with some reaching the periplasm as many as 240 minutes later than others (Fig. 1A, B). We hypothesized that as G. bimaculatus nuclei move through the cytoplasm, they preferentially move into nearby unoccupied space, which might explain the diversity of traveled paths.

To test this hypothesis, we binned nuclei into those that are in the periplasm and those that are not (Fig. $3 \mathrm{H} \mathrm{bottom}$ right). Then, for the nuclei in each subset we calculated a "space-seeking score", defined as the correlation of a nucleus's instantaneous movement vector with the vector that is oriented toward the most open space relative to the positions of its neighbors in $3 \mathrm{D}$ space (schematized in Fig. $3 \mathrm{H}$, top right; see details of this calculation in the Supplemental Information). We found that when they are not in the periplasm, Phase A nuclei tend to move into nearby open space (median space-seeking score $=0.61 ;$ Fig. $3 \mathrm{H}$ ), whereas Phase B nuclei do not (median space-seeking score $=0.12$; Fig. $3 \mathrm{H})$. Once nuclei reach the periplasm, however, they do not tend to move into open space during either Phase $\mathrm{A}$ or Phase $\mathrm{B}$ (median space-seeking score $=-0.21$ and -0.10 , respectively; Fig. $3 \mathrm{H}$ ). Consequently, they remain in the periplasm once they arrive there, forming the blastoderm, rather than moving back into the central yolk mass. We concluded that except for when they are in the periplasm, Phase A nuclei move preferentially into nearby unoccupied space.

\section{A simulation framework of cricket syncytial development}

We asked whether the nuclear movements during G. bimaculatus blastoderm formation could be explained by models that had been previously proposed for nuclear movement in other contexts. We considered three such candidate models of nuclear movement: (1) Cytoplasmic flows that move nuclei ${ }^{18,19}$; (2) Mutual, repulsive, active forces among nuclei ${ }^{21,52}$; (3) Local, asymmetric, active pulling forces on each nucleus ${ }^{20,38-41}$.

These modes of movement are not necessarily mutually exclusive ${ }^{14}$, yet for the sake of computational tractability, we assessed each of them in turn. The results presented in Fig. 1C and D contradicted the cytoplasmic flow model (1), so we did not consider it further. Similarly, we found that the empirical data contradicted the mutual repulsion model (2). Specifically, in models with mutual repulsion of nuclei, the magnitude of repulsion is highest when nuclei are closest to one another and it attenuates with increased distance ${ }^{26,40}$. Assuming that a nucleus's speed is directly related to the magnitude of the net force it experiences predicts that a nucleus should display its lowest speed when it is at the lowest density, i.e. when it is maximally distant from other nuclei. This is the opposite of what we observed in G. bimaculatus (Fig. 3E). In D. melanogaster, however, recent work on the spacing among nuclei after syncytial blastoderm formation led to models of mutual nuclear repulsion ${ }^{26,53}$. To further evaluate the possibility of mutual nuclear repulsion in forming the G. bimaculatus blastoderm (2), we selected one of these models (that of Dutta and colleagues $^{26}$ ) adapted it to $3 \mathrm{D}$, and implemented it in a simulation framework of preblastoderm syncytial nuclear movements in G. bimaculatus. We found that it did not produce a negative relationship between nuclear density and speed at any of the parameter settings we assessed (additional details are included in the Supplemental Information). Therefore we did not consider mutual repulsion further, and instead developed a simplified geometric model of a local, asymmetric, active pulling force on each nucleus (3). Below we summarize the key features and assumptions of the model. We describe the complete details of its computational implementation in the Supplemental Materials. Our model was inspired by research on nuclear movements in the red bread mold Neurospora crassa, the filamentous 
fungus Aspergillus nidulans, the nematode worm Caenorbabditis elegans, and D. melanogaster, which presented evidence for pulling forces on astral MTs and their associated microtubule-organizing center (MTOC) on each nucleus $^{20,38,39,41,54,55}$. In the present study, without specific empirical data on the cytoskeletal structures in the $G$. bimaculatus embryo, we abstracted the MT aster and MTOC contributions to the model as a "cloud" and "cloud origin”, respectively (schematized in Fig. $4 \mathrm{~A}$ ). We posited that, as in other systems $s^{38,54,56,57}$, cytoplasmic dyneins or functionally analogous molecules exert pulling forces on the astral MTs. We considered the dyneins to be uniformly distributed in the cytoplasm, and implemented their pulling forces as tugs on each voxel on the surface of each cloud. We divided each tug by a factor of $R^{2}$ where $R$ represented the distance from the voxel to its cloud origin. Scaling the relative strengths of the forces in this manner is analogous to a set of rods emanating from a point on the nucleus, normalized such that the total pull on a rod was proportional to its length, similar to previous work on $C$. elegans nucleus movement ${ }^{58}$. The sum of all the forces on a cloud origin causes nuclei to move through space and/or rotate. Larger and more asymmetric clouds therefore apply a relatively larger net pulling force (schematized in Fig. 4A). To assign a magnitude to the pulling forces, we calibrated by fitting a constant factor such that the maximum speed of simulated nuclei matches that of the nuclei in a real embryo. Similarly, we set the maximum cloud radius by fitting the simulated nuclear density-speed relationship to that of the real embryos. The clouds regrow after each division, but their growth is occluded by one another and by the internal surface of the simulated eggshell (Fig. 4B). If simulated nuclei proliferate in a limited volume, local density increases, which means that over time, clouds grow smaller (Fig. 4C, D).

The first goal of the simulation was to determine whether pulling clouds could collectively generate the observed density-dependent speeds and space-seeking behaviours without either of those phenomena being encoded explicitly. The second goal was to determine whether the overall spatial and temporal patterns of G. bimaculatus nuclear movement could be recapitulated with a minimal set of hard-coded parameters. Therefore, in the simulation, we matched the overall embryo morphology, division cycle distribution, and bias towards the periplasm to those of the empirical embryo. Each nucleus cycles from a Phase A state to a Phase B state, then it divides and returns to Phase A. During Phase A, the nucleus's cloud grows to its maximum size (unless spatially constrained) and exerts pulling forces on the nucleus. During Phase B, the cloud is absent. In both Phase A and Phase B, we added a small random contribution to nuclear movement by drawing from a uniform distribution with a mean random step size of $0.017 \mu \mathrm{m}$ per minute. To assign a mitotic cycle duration to each nucleus, we sampled directly from the empirical relationship between density and cycle duration, determined by a linear fit to the data $($ slope $=11.18$, intercept $=$ -111.85). We added an attraction toward the periplasm of the simulated embryo, the magnitude of which was tuned to match the rate at which nuclei moved towards the periplasm in empirical data (this attraction was $25 \%$ of the magnitude of the net cloud-pulling force on an isolated nucleus). The model does not include terms for viscosity of the cytoplasm, fluid flows, pushing forces of any kind, or maternally provided signals in the yolk.

\section{Simulation results}

We found that the active pulling model qualitatively and quantitatively recapitulates many features of G. bimaculatus syncytial blastoderm formation. Simulated nuclei spread out through the embryo (Fig. 4E), occupying the space along the A-P axis in a spatiotemporal pattern like that of real embryos (Fig. 4F, G). Simulated embryo-wide speed oscillations are likewise similar to empirical oscillations (Fig. 4H). The cell cycle and density-dependent nuclear speeds are also recapitulated (Fig. 4I-K). As in Fig. 3E and F, we fitted a curve to the speed vs. nuclear density relationship, which yielded density scales for Phases A and B of 26.75 density units (90\% CI: 24.2 to 29.3) and 12108 density units (90\% CI: 11124 to 13092), respectively. Nuclei in the simulated embryo, like those in real embryos, exhibit a negative relationship between density and speed in Phase A (Fig. 4J). In Phase B, with no pulling clouds, nuclear speed does not vary with density at all (Fig. 4K). Last, we assessed the tendency to move into unoccupied space. As in real nuclei, simulated nuclei that are not in the periplasm have a tendency to move into unoccupied space in Phase A (median space-seeking score $=0.86$ ) but not during Phase B (median space-seeking score = -0.04; Fig. $4 \mathrm{~K}$ ). Once simulated nuclei reach the periplasm, they tend to move into nearby open space during Phase $\mathrm{A}$, 
unlike those of real embryos (median space-seeking score $=0.75$; compare Fig. $4 \mathrm{~K}$ to Fig. $3 \mathrm{~F}$ ). In D. melanogaster there is a cellular mechanism that holds nuclei in place once they reach the periplasm ${ }^{16,48-51}$. We suggest that there is likely a similar mechanism at work in G. bimaculatus that has not been included in our model. In light of the simulation results, we concluded that a local, asymmetric, active pulling force on each nucleus is consistent with most of the observed nuclear behaviours in G. bimaculatus.

\section{Constricting embryos}

Based on the empirical data and simulation results, we hypothesized that nuclear density determines nuclear speed, and that the negative relationship between density and speed emerges from an asymmetric pulling mechanism. With the descriptive data and simulations alone, however, we could not rule out the possibility that the observed changes in nuclear speed were caused by a spatially localized or temporally varying signal in the cytoplasm. Therefore, to test our hypothesis and assess the alternatives, we experimentally altered the density of nuclei, accomplished by physically manipulating the geometry of embryos. We designed and built a device to constrict an embryo from the outside by wrapping a human hair around it and incrementally increasing the tension on the hair. Specifically, we constricted $G$. bimaculatus embryos width-wise at the beginning of syncytial development, and then mounted them in a glass bottom dish for epifluorescence microscopy (Fig. 5A; see Supplemental Information for detailed methods and for the design file of the device's components). With this mode of microscopy, we collected 2D+T datasets, imaging nuclei through approximately one-third of the $z$ depth of the embryo. This imaging modality necessitated 2D measures of speed and density. Moreover, those measures and the total nucleus counts were not comparable on an absolute scale to the equivalent values in the $3 \mathrm{D}+\mathrm{T}$ datasets. Thus, for these analyses, we compared between and within embryos that were all imaged using epifluorescence microscopy. The first zygotic nuclear division typically occurs approximately $60 \%$ from the anterior pole along the length of the embryo. We constricted embryos at a position $25 \%$ to $35 \%$ from the anterior pole (Fig. 5A). By pinching a region down to approximately one-third of the radius of the embryo, we generated two pseudo-compartments in the embryo, each of which was smaller than an unconstricted embryo (Fig. 5B). In both pseudo-compartments of the constricted embryos, the patterns of nuclear density over time and space differed markedly from those of unmanipulated embryos, which enabled us to decouple nuclear speed and density from any as-yet undetected spatially localized cytoplasmic determinants.

First, we compared nuclear behaviour between posteriors of constricted embryos to unconstricted embryos. In effect, this allowed us to control for A-P position and developmental time while changing nuclear density. To compare the data from multiple embryos on a single plot, we stage-matched datasets $(\mathrm{n}=3$ embryos per treatment) by the total number of nuclei in each embryo as a proxy for developmental time. As nuclei divide and move within a constricted posterior volume, the total space available to them is reduced compared to an unmanipulated embryo, which causes them to experience higher densities earlier in development than they would otherwise (Fig. 5C). For instance, when there are 200 total nuclei, median density is 20.1 density units (25th percentile $=18.1$, 75th percentile $=22.2$ ) in constricted embryos and 15.2 density units (25th percentile $=11,75$ th percentile $=19.5$ ) in unconstricted embryos. The higher density nuclei in constricted embryos move more slowly than those in stage-matched unmanipulated embryos where the density was lower (Fig. 5D). At 200 total nuclei, median speed was $0.68 \mu \mathrm{m}$ per minute (25th percentile $=0.57,75$ th percentile $=0.73)$ in constricted embryos and $0.93 \mu \mathrm{m}$ per minute $(25$ th percentile $=$ $0.85,75$ th percentile $=1.06$ ) in unconstricted embryos. Last, we computed all instantaneous nuclear speeds and densities in each constricted posterior and unconstricted dataset, finding that nuclei follow the same density-speed relationship in constricted and unconstricted embryos (Fig. 5E). These results are consistent with a mechanism where speed is determined by nuclear density, rather than A-P position or developmental time.

The constricted embryos provided the opportunity to analyze nuclei not only under ectopically high local nuclear densities, but also under ectopically low densities. The latter becomes possible when, in constricted embryos, some nuclei traverse the constricted region and populate the formerly unoccupied pseudo-compartment (Fig. 5A). These nuclei move into a low density region from a comparatively high density region, creating an abrupt change in local 
density for a subset of nuclei. With this experiment, we altered the developmental time-course of densities that the nuclei experienced. We quantified the nuclear speeds under these conditions, and plotted nuclear speed vs. time for the posterior (ectopically high density) and anterior (ectopically low density) subsets of nuclei (Fig. 5F). We observed that posterior nuclei undergo speed oscillations and overall decreasing speeds, similar to nuclei in unperturbed embryos. We also observed that 50 to 80 minutes after the start of each dataset, nuclei emerge from the constriction (indicated by dotted lines; Fig. 5F), whereupon they speed up as they populate the unoccupied space, slowing down again once local nuclear density increases, ultimately coming to match the speeds of the nuclei in the posterior (compare magenta to orange speed traces; Fig. 5F). We interpreted these results as further evidence that nuclear speed is determined by density, independent of developmental time or spatial location.

\section{Simulating blastoderm formation for embryos with other shapes}

We wanted to know whether our model, parameterized and validated on unmanipulated G. bimaculatus, could successfully predict nuclear behaviours in a constricted embryo. We used the same simulation procedure described above, changing only the geometry of the embryo to a constricted shape (Fig. 5G, H). The simulated pulling clouds completely fill the posterior compartment before any nuclei emerge through the constriction (Fig. 5G). This results in a distribution of nuclei over time in the simulations that is qualitatively similar to that of the real constricted embryos (Fig. 5H; compare to 5A). As in the real embryos, the simulated nuclei also exhibit speed oscillations that get slower and of smaller amplitude over time in each pseudo-compartment, but that pattern is offset in time and space for nuclei in the anterior, which abruptly speed up once they pass through the constriction (Fig. $5 \mathrm{H})$. We concluded that simulations broadly recapitulated the experimental results from an atypical embryo geometry, and interpreted this as further support for a model of local, asymmetric, active pulling forces on G. bimaculatus syncytial nuclei.

With the aim of uncovering mechanistic principles that might extend beyond this specific case, we asked whether this predictive model of nuclear behavior, derived from observations on G. bimaculatus, could be generalized to describe axial expansion in the syncytial embryos of other insects. As a first step in this direction, we asked how well the parameterized model would perform at simulating blastoderm formation in insect species that are either closely or distantly related to G. bimaculatus. The locust Schistocerca gregaria is an orthopteran insect, like G. Bimaculatus, but lays eggs that are $2.5 \times$ longer and $1.5 \times$ wider than $G$. bimaculatus eggs ${ }^{34,59}$. The first nuclear division in $S$. gregaria also occurs closer to the posterior pole than that of $G$. bimaculatus ${ }^{59-61}$. We deployed the computational model described earlier in the text, leaving all parameters the same except for two changes: (1) the morphology of the embryo was set to be an ellipsoid with the volume and approximate shape of the $S$. gregaria embryo ${ }^{59}$; (2) the position of the first division was set to $85 \%$ from the anterior pole along the length of the embryo, similar to that of S. gregaria ${ }^{59}$. Based on comparisons to previous work that used fixed embryo preparations, and acknowledging differences in imaging modality, we detected suggestive similarities between the features of nuclear positioning in the simulated embryo and in real locust embryos ${ }^{59}$ (Fig. 6A, B). Specifically, simulated nuclei reach the posterior pole of the embryo while the anterior two-thirds of the embryo are still devoid of nuclei. Simulated nuclei also form a gradient in their spacing, with the largest internuclear distances at the anterior of the expanding front of nuclei (compare Fig. 6A and B). Given these similarities, we hypothesize that a mechanism of local, asymmetric, active pulling forces also operates in the S. gregaria preblastoderm embryo.

By contrast, our parameterized model run with a $D$. melanogaster embryo morphology and first division location, produces arrangements of nuclei that differ qualitatively from those of real $D$. melanogaster embryos. The simulated nuclei spread throughout the volume of the embryo by moving in all directions, with some moving into the periplasm before the rest (Fig. 6C). In real embryos, nuclei spread out predominantly along the A-P axis without moving into the periplasm, then form an ordered shell-like arrangement ${ }^{19,21}$ (Fig. 6D), followed by simultaneous movements into the periplasm ${ }^{21,22}$ (not shown). We interpret this result as evidence against the hypothesis that there is a shared cellular mechanism that scales with embryo size to generate preblastoderm nuclear behaviour across insect taxa ${ }^{20}$. 
This result is also consistent with recent work on $D$. melanogaster, which demonstrated that cytoplasmic flows generate some of the preblastoderm nuclear movements ${ }^{19}$. In the future it will be fruitful to use experimentally validated computational models to develop hypotheses for nuclear dynamics in other poorly studied systems.

\section{Conclusions}

Our computational model was inspired by empirical descriptions of astral MTs and nuclear movements in other contexts $^{1,20,38,41}$. Given the model's effectiveness in capturing G. bimaculatus nuclear dynamics, we speculate that cytoplasmic dyneins interacting with astral MTs may indeed be the most likely molecular cause of the asymmetric pulling forces on $G$. bimaculatus syncytial nuclei. There is evidence that such a mechanism may also be present in D. melanogaster embryos but obscured by the comparatively dramatic effect of cytoplasmic flows ${ }^{19}$ : nuclei in non-flowing cytoplasmic extracts of preblastoderm $D$. melanogaster embryos move apart from one another in a MTand centrosome-dependent manner that appears to be consistent with a pulling force on asters ${ }^{20}$. Alternatively, asymmetric active forces in G. bimaculatus could be generated by a molecular mechanism that does not involve dynein and astral MTs ${ }^{40}$. One possibility is that dynamic remodeling of the actin cytoskeleton in the immediate neighborhood of a nucleus could generate nucleus movements by local, asymmetric fluidization ${ }^{17}$. Another is that asymmetric contractile interactions within an actomyosin network at the periphery of each energid could pull an energid - and the nucleus embedded within it—-through the rest of the cytoplasm. It is also possible that MTs and actomyosin contractility jointly contribute to nuclear movements ${ }^{14,17,62}$.

The results of the present study enable us to make inferences about cytoplasmic signals that may regulate the cell cycle behaviours of nuclei during G. bimaculatus syncytial development. Similar to D. melanogaster ${ }^{20}, G$. bimaculatus nuclei speed up after each division and then slow down, which suggests that the mechanism driving nuclear movement is coupled to the cell cycle. In D. melanogaster, changes in the localization and activity of cytoplasmic Cdk1 and CycB over the course of the cell cycle affect MT and actomyosin dynamics ${ }^{18,19,63}$. We hypothesize that the nucleus-moving mechanism in G. bimaculatus is affected in a similar, cell cycle-coupled manner. Unlike D. melanogaster $20,22,47,64$, however, G. bimaculatus cycle duration appears to be locally responsive to nuclear density, rather than coordinated throughout the entire embryo. It has been shown that experimentally increased levels of $\mathrm{CycB}$ protein reduce the duration of interphase in D. melanogaster ${ }^{47}$, and that $\mathrm{CycB}$ is degraded at a subcellular spatial scale ${ }^{65}$. Therefore, one possibility is that in $G$. bimaculatus, regions with relatively high nuclear density locally reduce the levels of $\mathrm{CycB}$ (or a protein with an analogous function), which causes the duration of interphase to increase accordingly. If true, diffusion would not be predicted to generate uniform cytoplasmic protein levels on relevant time scales in the much larger $G$. bimaculatus embryos. In general, such a mechanism would be consistent with the comparatively heterogeneous cell cycle durations in G. bimaculatus.

Our results have implications for the mechanisms governing subsequent G. bimaculatus development. Researchers have begun to describe some of the earliest patterning events in G. bimaculatus, including aspects of the establishment of the A-P axis, the dorsal-ventral axis, and the bipartition of the blastodermal cells into embryonic and extraembryonic tissues ${ }^{35,66-68}$. The present study demonstrates that the early G. bimaculatus embryo does not have stereotypic divisions nor movements, but instead each nucleus's behaviour is determined by the geometry of its neighbors. Collectively, these stochastic interactions, constrained by local rules, generate an emergent uniform spacing of blastodermal nuclei across the embryo. Accordingly, we hypothesize that any axial patterning information is likely to be "stored" stored in the central cytoplasmic mass or in the periplasm, and that any such putative localized signals do not have a detectable effect on syncytial nuclear behaviour. This hypothesis predicts that the subdivision of the blastoderm into the embryonic anlage and extraembryonic tissues ${ }^{68}$ is not determined during the preblastoderm stage, and that the two populations of cells only become separate lineages after a blastoderm has formed.

The computational model we present for blastoderm formation enables us to make predictions about early embryogenesis in other insect species. Insect eggs have a wide range of shapes and $\operatorname{sizes}^{69,70}$, and the egg determines 
the shape and size of the syncytial embryo ${ }^{10,11}$. In our simulations, embryo morphology-as well as the location of the initial zygotic division-plays a major role in determining patterns of nuclear movement over the course of blastoderm formation. If an asymmetric pulling mechanism drives syncytial nuclear movements in other insect species, we predict that patterns of syncytial nuclear behaviour will tend to co-vary predictably with egg morphology. For instance, in an embryo with a higher aspect ratio ${ }^{69,70}$ we would predict a front of low-density nuclei moving at relatively high speeds into unoccupied regions of the embryo, trailed by nuclei arranged in a density gradient (and exhibiting concomitant speeds and cell cycle durations). In a spherical embryo (i.e. an aspect ratio of 1) with a centrally located first zygotic nucleus, we would expect comparatively uniform movements and densities over time and space. In smaller embryos, nuclei would reach an equilibrium spacing more quickly than in larger embryos. These predictions can be tested in a straightforward manner by comparing timed and fixed embryo preparations from insect species that are closely related, yet have embryos of different sizes and/or shapes.

We suggest that elements of the empirical and modeling approaches in the current study could also be fruitfully applied to better understand nuclear behaviours in other multinucleate cell types. In mammalian muscle cells, there are numerous types of nuclear movement, with distinct cellular mechanisms implicated in each one ${ }^{4,71,72}$. Likewise, in filamentous fungi, nuclei are moved by several mechanisms, with important roles for cytoplasmic dynein and astral $\mathrm{MTs}^{3,73}$. Arbuscular mycorrhizal fungi have multinucleate spores with hundreds of nuclei, organized in a shared volume, but the cellular mechanisms underlying this arrangement are not known ${ }^{74,75}$. It will be illuminating to discover the ways in which MT- and F-actin-based molecular mechanisms are deployed in different distantly related species to generate forces on nuclei, moving them into the proper arrangement at the right time and place. Comparing such mechanisms across taxa will provide insight into how the conserved eukaryotic cellular machinery shapes developmental evolution.

\section{Acknowledgements}

This work was supported by National Science Foundation (NSF) award IOS-1257217 to CGE, a US Department of Energy (DOE) Computational Science Graduate Fellowship JH, a National Science Foundation Graduate Training Fellowship to SD, and the Applied Mathematics Program of the US DOE Office of Advanced Scientific Computing Research under Contract DE-AC02-05CH11231 to CHR. TN was supported by a JSPS Overseas Research Fellowship (Received Number 693) from the Japan Society for the Promotion of Science. We thank the Extavour and Rycroft lab members, the Harvard Center for Biological Imaging, the NSF-Simons Center for Mathematical and Statistical Analysis of Biology at Harvard University, supported by NSF Grant DMS-1764269, and the Harvard Faculty of Arts and Sciences Quantitative Biology Initiative for discussion and support. We thank Rakeyah Ahsan for assistance with animal culture.

\section{Author contributions}

SD and CGE conceived of the project; SD and TN performed all transgenic and imaging experiments; JH and CHR designed and implemented all mathematical models in consultation with SD, TN and CGE; all authors contributed to writing of the MS. 
bioRxiv preprint doi: https://doi.org/10.1101/2021.04.26.441395; this version posted April 28, 2021. The copyright holder for this preprint (which was not certified by peer review) is the author/funder, who has granted bioRxiv a license to display the preprint in perpetuity. It is made available under aCC-BY-NC-ND 4.0 International license.

A

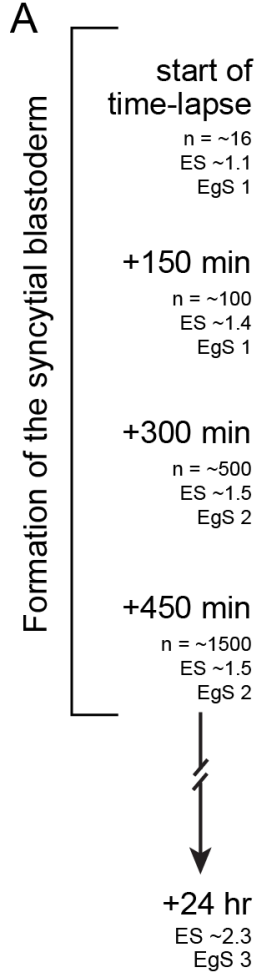

B

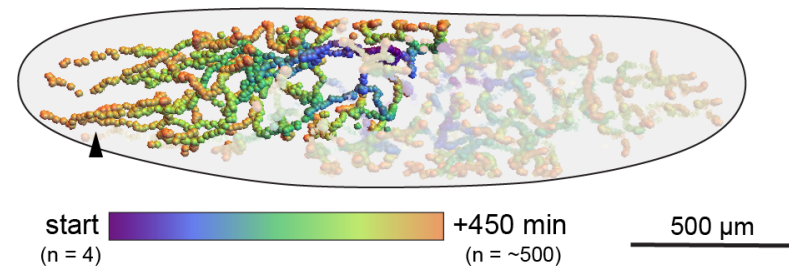

Cellularization \& embryonic rudiment formation rudiment
C

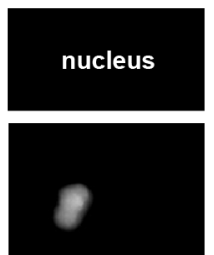

nucleus

energid

cytoplasm
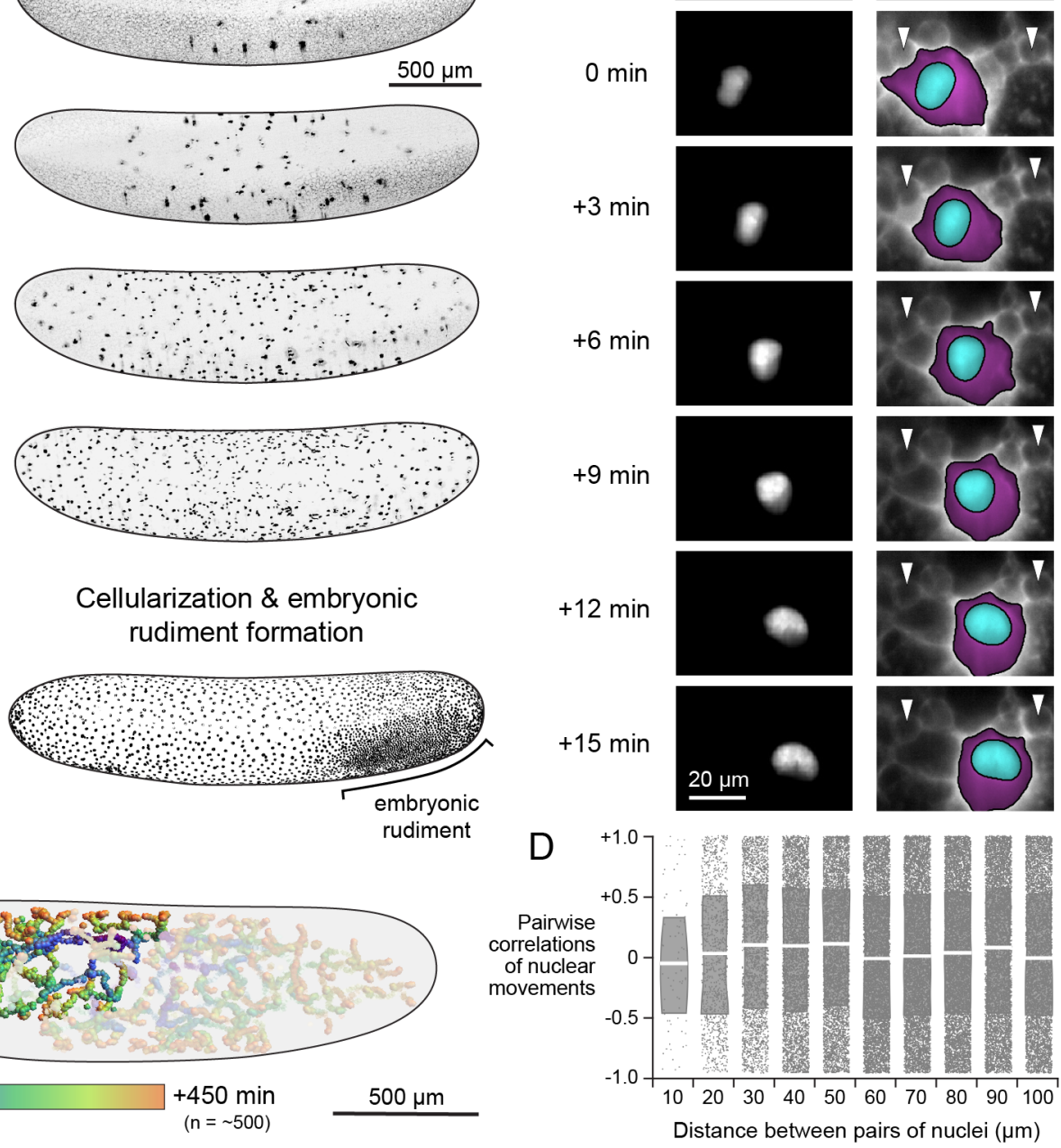

Figure 1: Overview of G. bimaculatus blastoderm formation and evidence that nuclei move actively, rather than being moved along by flowing cytoplasm. A, Time points from the embryonic syncytial development of G. bimaculatus, displayed as $z$-projections of 3D stacks. Micrographs are from an H2B-EGFP transgenic G. bimaculatus embryo live-imaged using a lightsheet microscope over eight hours of development at $28.5^{\circ} \mathrm{C}$, capturing nuclear divisions and movements throughout the syncytial embryo. The nuclei (n) arrange into a single layer, after which cellularization occurs and the embryonic rudiment forms. Embryos are oriented laterally with ventral to the bottom and anterior to the left. Anterior is to the left in all subsequent figures. B, Nuclei were tracked to produce a $3 \mathrm{D}+\mathrm{T}$ dataset of nuclear lineages. All nuclear tracks are displayed for an example embryo, with the lineage descended from a single nucleus highlighted. Color scale represents time; $\mathrm{n}=$ number of nuclei. Black arrowhead highlights nuclei that move in a highly directed manner toward the anterior pole. C, Example time points from a 3D+T dataset of a transgenic G. bimaculatus embryo with nuclei and cytoplasm fluorescently marked (further details in Methods). Left column shows the nucleus channel and the right column shows the cytoplasm channel, with the energid cytoplasm highlighted in magenta and the nucleus highlighted in cyan. White arrowheads mark two different yolk granules that remain in place as the nucleus moves past them. $\mathrm{D}$, Pairwise correlations between the instantaneous movement vectors of pairs of non-sister nuclei. White line indicates median and box indicates interquartile range. 
bioRxiv preprint doi: https://doi.org/10.1101/2021.04.26.441395; this version posted April 28, 2021. The copyright holder for this preprint (which was not certified by peer review) is the author/funder, who has granted bioRxiv a license to display the preprint in perpetuity. It is made available under aCC-BY-NC-ND 4.0 International license.
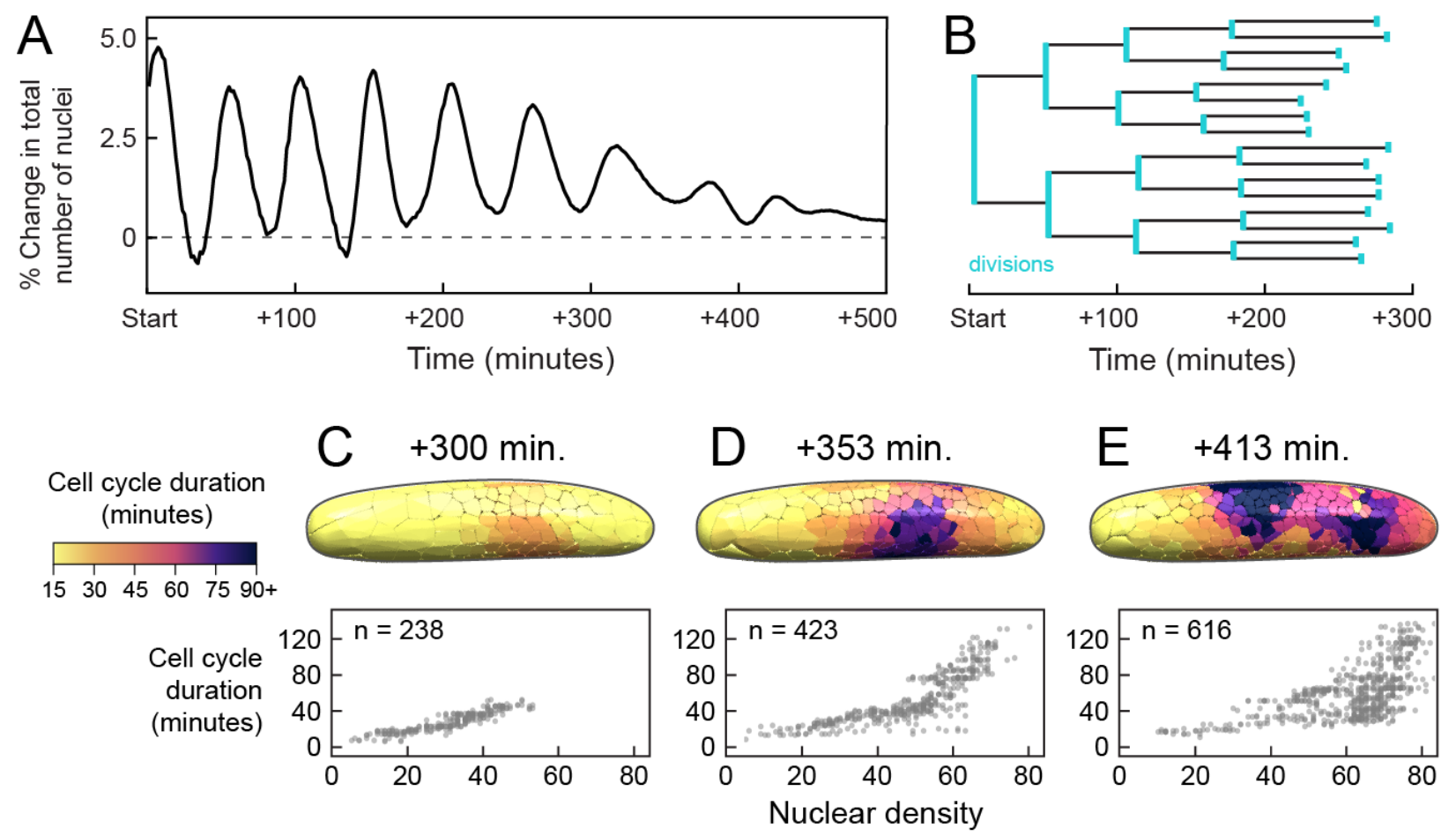

Figure 2: Mitotic cycle duration covaries with nuclear density, explaining a marked decline in division synchronicity. A, Percent change in the total number of observable nuclei over time. Divisions become increasingly asynchronous over the course of syncytial development. B, Example lineage of dividing nuclei, starting with one of the nuclei at the four nucleus stage with divisions marked in turquoise. Over four division cycles, mean cycle duration increased from 49 to 87 minutes. C-E, Cell cycle duration was positively associated with local density. Cell cycle duration was calculated in the vicinity of each nucleus by measuring the time elapsed until the number of nuclei within a $150 \mu \mathrm{m}$ radius increased by $25 \%$. Top row shows three example time points, with local cell cycle duration displayed as colored volumes, each of which contains a single nucleus. Bottom row shows scatterplots of the local cell cycle duration times and nuclear densities at each time point; $\mathrm{n}=$ number of nuclei. 
bioRxiv preprint doi: https://doi.org/10.1101/2021.04.26.441395; this version posted April 28, 2021. The copyright holder for this preprint (which was not certified by peer review) is the author/funder, who has granted bioRxiv a license to display the preprint in perpetuity. It is made available under aCC-BY-NC-ND 4.0 International license.
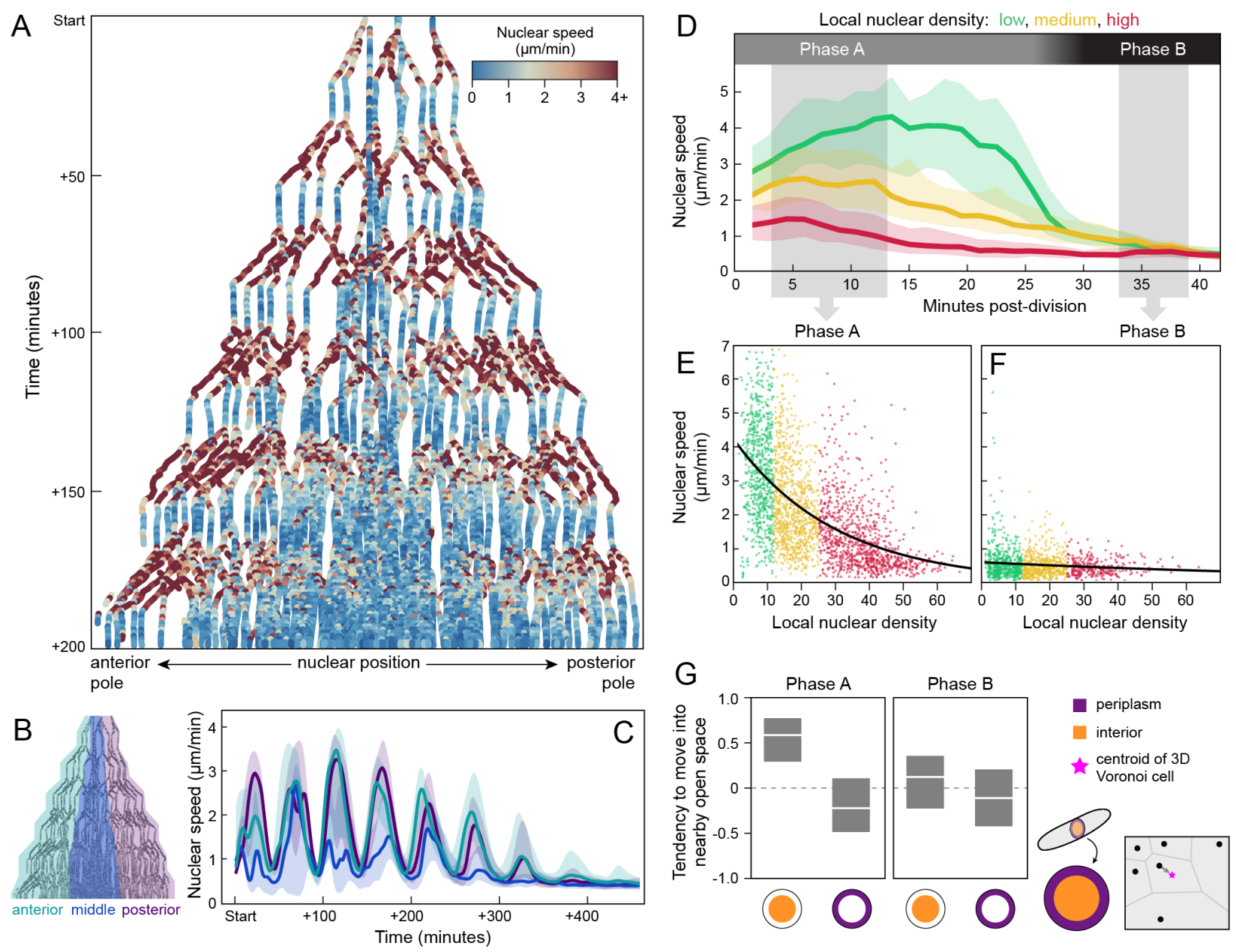

Figure 3: After each division, nuclear speed covaries with local nuclear density, and nuclei move into nearby open space. A, Positions of all nuclei along anterior-posterior axis $(x)$, plotted over 200 minutes of syncytial development $(y)$. Each dot represents a single nucleus at a single time point, colored according to its instantaneous speed. The fastest nuclei are shown in red and the slowest in blue. The first zygotic nuclear division occurred approximately $60 \%$ from the anterior pole of the embryo. Imaging began after the second nuclear division cycle. Nuclei underwent roughly synchronous speed oscillations, visible as alternating bands of blue and red. B, Schematic of nuclei partitioned into three terciles of equal numbers of nuclei: anterior, middle, and posterior (turquoise, dark blue, and purple, respectively). C, Compared to the anterior and posterior poles, speed oscillations dissipate earliest in the middle third of the embryo, where local nuclear density is highest. Center line represents median and shaded regions represent 25 th to 75 th percentile of the data. D-F, Nuclei from all time points and all regions of the embryo were grouped into three bins, according to their local density, calculated as the number of nuclei within a $150-\mu$ m radius ("low": $<11$ nuclei, "medium": $\geq 11$ and $<29$ nuclei, "high: $\geq 29$ nuclei; see SI for details). D, Nuclear speed traces after division. Plot depicts the movements after each mitosis concluded and daughter nuclei re-formed. Nuclei move relatively quickly after a division and then slow down. We refer to these periods as "Phase A" and "Phase B". The biphasic pattern is most pronounced for low density nuclei (green). Center line represents median and shaded regions represent 25th to 75th percentile of the data. E, F, The relationship between a nucleus's instantaneous speed and its local density. Data are shown from two periods of time post-division: $\mathrm{t}=3$ to $\mathrm{t}=13.5$ minutes $(\mathbf{E})$ and $t=33 \mathrm{~min}$ to $t=39 \mathrm{~min}$ (F). Black line represents a best-fit curve of the form $y=y_{0} e^{-x / x_{0}}$, which yields a density scale $\left(x_{0}\right)$ of 30.4 density units for Phase A (90\% CI: 28.8 to 32.1) and 120.4 density units for Phase B (90\% CI: 73.4 to 167.4). G, We calculated a nucleus's tendency to move into nearby open space as its movement vector's correlation with the vector from its current position to the centroid of its Voronoi cell (pink star; see SI for details). Nuclei were also subdivided into those in the "interior" of the embryo, or in the periplasm (defined here as within $75 \mu \mathrm{m}$ of the eggshell). Phase A nuclei in the interior tend to move into nearby open space, but not when they are in the periplasm (left plot). Phase B nuclei do not tend to move into nearby open space regardless of where they are (right plot). Center line represents median and boxes represent 25 th to 75 th percentile of the data. 
bioRxiv preprint doi: https://doi.org/10.1101/2021.04.26.441395; this version posted April 28, 2021. The copyright holder for this preprint (which was not certified by peer review) is the author/funder, who has granted bioRxiv a license to display the preprint in perpetuity. It is made available under aCC-BY-NC-ND 4.0 International license.

A

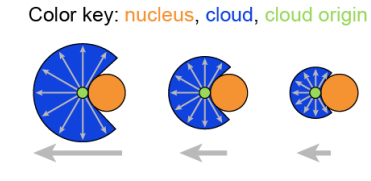

B

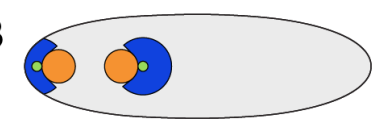

C
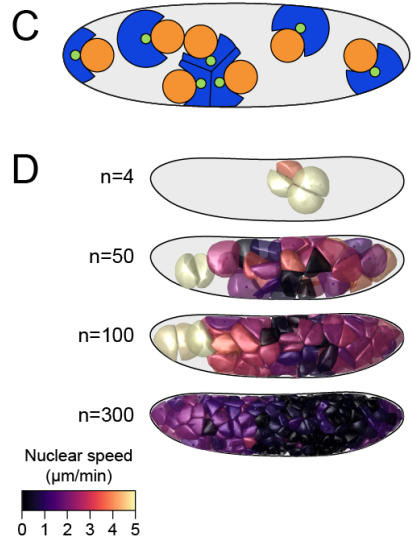

$\mathrm{E}$
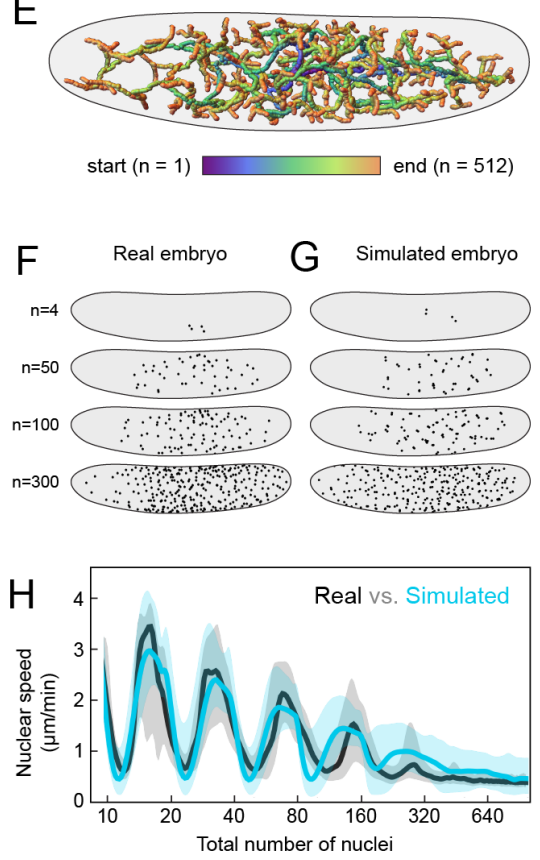
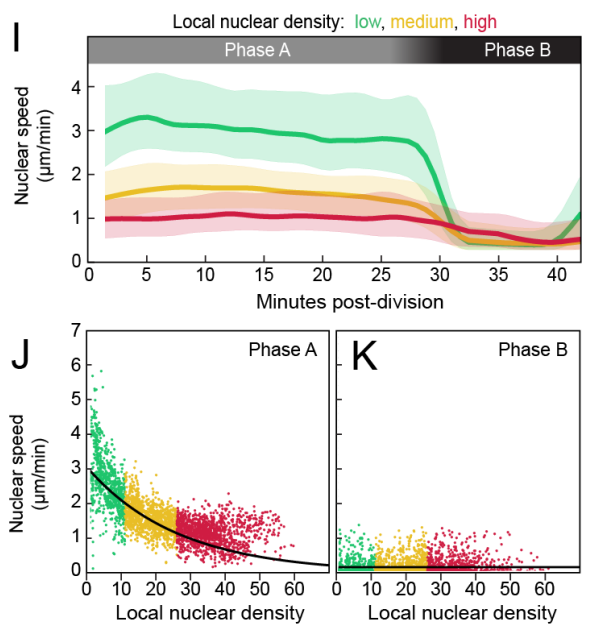

$\mathrm{L}$

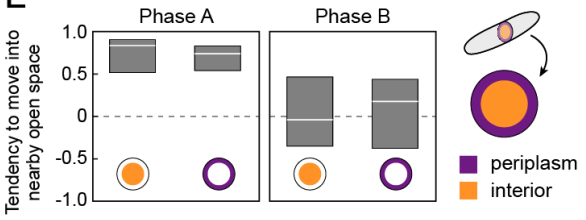

Figure 4: A simple model based on local pulling clouds recapitulate most features of G. bimaculatus syncytial development. A-C, Schematic representation of a computational method for simulating syncytial development. Nuclear movements were simulated in a 3D embryo, but it is schematized here in $2 \mathrm{D}$ (diagrams not to scale). See SI for further details on the method's implementation. A, Each nucleus (orange) moves due to a pull (grey arrow) from a "cloud" (blue) that grows from a "cloud origin" (green) on the nucleus to a fixed maximum radius. Each voxel within the cloud pulls on the nucleus via the origin point. The nucleus occludes symmetrical growth of the cloud, and as a consequence, the cloud is asymmetric and there is a net pull on the nucleus that is determined by the size and shape of the cloud. B, Before a nuclear division, the cloud's origin divides into two daughter origins, which are assigned random positions opposite one another on the surface of the nucleus. Then the nucleus divides, with each daughter nucleus inheriting a cloud origin, each of which in turn grows a new cloud. The eggshell occludes the growth of clouds. C, Nuclei continue to divide and spread. Growing clouds occlude one another, which means that as local nuclear density increases, clouds are unable to grow as large as under lower local densities. As a consequence, nuclear speed is lower in regions with higher nuclear density. The model also includes an overall bias in movement toward the periplasm (not shown). D, 3D renderings of pulling clouds from four selected time points of a simulation of syncytial blastoderm formation under this model. Each cloud is colored according to the instantaneous speed of its nucleus. E, 3D paths of simulated syncytial development, with nuclear positions tracked over time. F, G, $z$-projections of nuclear positions in example real $(\mathbf{F})$ and simulated $(\mathbf{G})$ time points, matched by total number of nuclei $(\mathrm{n})$. $\mathbf{H}$, Whole-embryo nuclear speed $(y)$ vs. total number of nuclei $(x, \log$ scale) from a simulated (turquoise) and real (black) embryo (shaded region represents 25 th to 75th percentiles). I-L, Simulated nuclei exhibit speeds and directions that co-vary with density and cycle phase, similar to the empirical patterns (compare to Fig. 3D-G; quantities were calculated as described above). I, Simulated nuclear speed traces after division. J, K, The relationship between a simulated nucleus's instantaneous speed and its local density. Time intervals represented in the Phase A and B panels are the same as those in Fig. 3E and F. Best fit density scale $\left(x_{0}\right)$ was 30.44 density units for Phase A (90\% CI: 28.78 to 32.11) and 120.38 density units for Phase B (90\% CI: 113 to 127). L, Simulated Phase A nuclei in the interior and periplasm tended to move into nearby open space (left plot). Simulated Phase B nuclei did not tend to move into nearby open space regardless of where they were (right plot). 
bioRxiv preprint doi: https://doi.org/10.1101/2021.04.26.441395; this version posted April 28, 2021. The copyright holder for this preprint (which was not certified by peer review) is the author/funder, who has granted bioRxiv a license to display the preprint in perpetuity. It is made available under aCC-BY-NC-ND 4.0 International license.

A

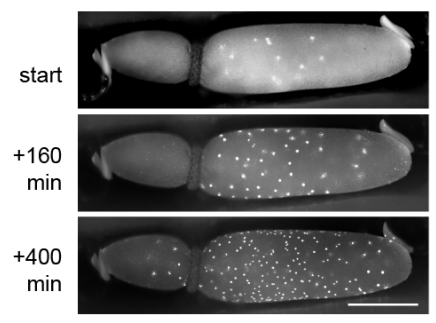

$\mathrm{B}$

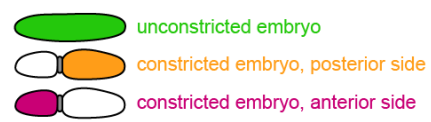

C

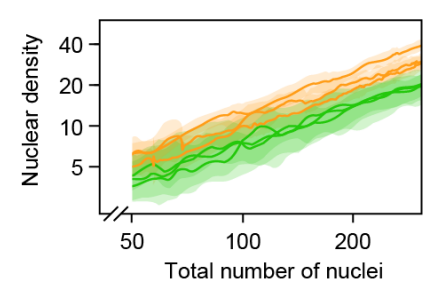

$\mathrm{D}$

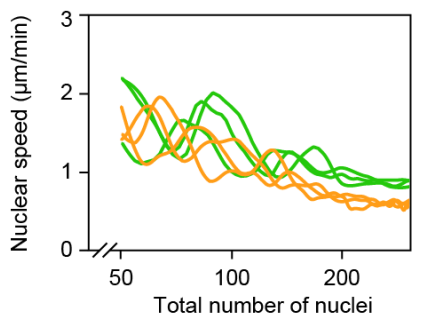

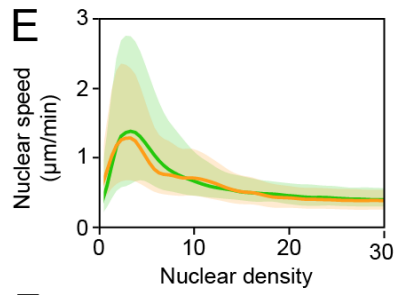
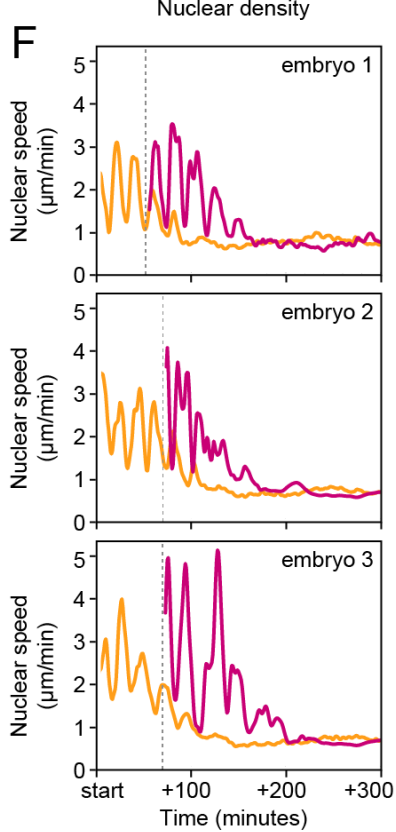

G

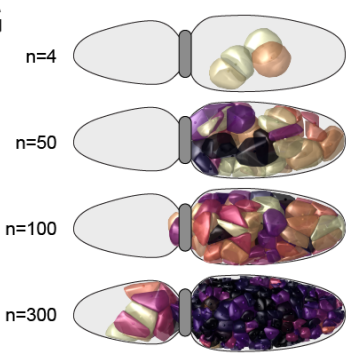

$\mathrm{H}$
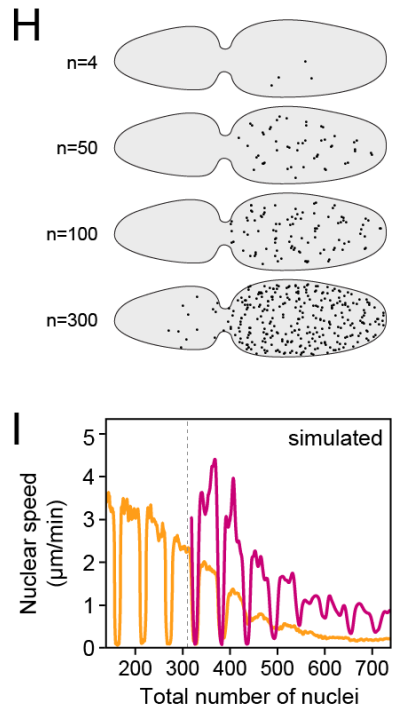

Figure 5: Embryo constrictions demonstrate that nuclear density, rather than spatially or temporally localized signals, determines the speed of nuclear movements. A, Three time points of an embryo that was constricted with a human hair (see SI and Methods), imaged with epifluorescence microscopy. Scale bar represents $500 \mu \mathrm{m}$. B, Color key and schematic for subsets of nuclei represented in this figure. $\mathbf{C}$, Nuclear density $(y, \log$ scale) plotted against total number of nuclei $(x, \log$ scale) for unconstricted embryos (green; $n=3$ ) and the posterior side of constricted embryos (orange; $n=3$ ). Center line represents median and shaded regions represent 25 th to 75 th percentile of each embryo's data. Constrictions caused higher nuclear densities for a given number of total nuclei. $\mathbf{D}$, Median nuclear speed $(y)$ vs. total number of nuclei $(x, \log$ scale) for the same datasets as in C. As the posterior side of constricted embryos fill with nuclei, local densities increase and nuclear speeds decrease, as compared to unconstricted embryos. E, Nuclear speed $(y)$ as a function of nuclear density $(x)$ for all instantaneous nuclear movements across the datasets shown in $\mathbf{C}$ and $\mathbf{D}$. Embryonic constrictions do not change the relationship between density and speed. Center line represents median and shaded regions represent 25 th to 75 th percentile of the data. F, Median nuclear speed $(y)$ over time $(x)$ for three example constricted embryos. Posterior and anterior nuclei are shown in orange and magenta, respectively. Vertical dotted line indicates the time point when nuclei first emerge from the constriction into the anterior side. When nuclei pass through the constriction into the anterior side of the constriction, they speed up as they populate the unoccupied compartment of the embryo, slowing down again once local nuclear density increases. G-I, Our model of pulling clouds (Fig. 4A) qualitatively recapitulated empirical nuclear behaviours in a simulated constricted embryo. G, 3D renderings of pulling clouds from four selected time points of a simulation of syncytial blastoderm formation in a constricted embryo. Each cloud is colored according to the instantaneous speed of its nucleus, following the colormap in Fig. 4D. H, $z$-projections of nuclear positions in a simulated constricted embryo. I, Median nuclear speed $(y)$ vs. total number of nuclei $(x)$ for a simulated constricted embryo, plotted as in $\mathbf{F}$. 
bioRxiv preprint doi: https://doi.org/10.1101/2021.04.26.441395; this version posted April 28, 2021. The copyright holder for this preprint (which was not certified by peer review) is the author/funder, who has granted bioRxiv a license to display the preprint in perpetuity. It is made available under aCC-BY-NC-ND 4.0 International license.

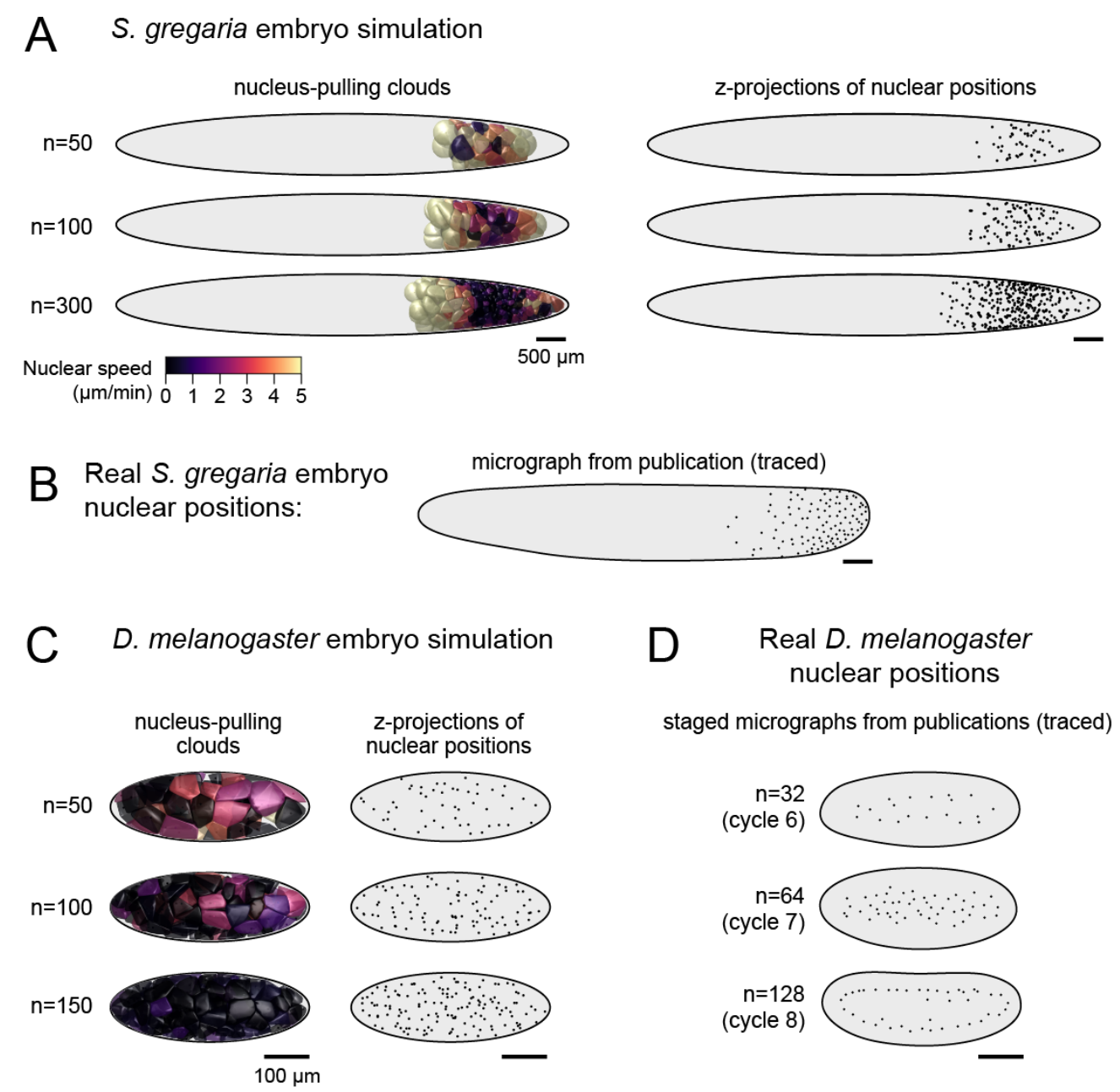

Figure 6: The parameterized model of local pulling clouds qualitatively recapitulates blastoderm formation of another orthopteran, but not $D$. melanogaster. A, Selected time points of simulated blastoderm formation in an ellipsoid with the length and width of the locust Schistocerca gregaria embryo. The location of the first division was set to $85 \%$ from the anterior pole along the length of the embryo, similar to that of S. gregaria $^{59}$. Left column: 3D renderings of pulling clouds, colored by instantaneous speed. Right column: $z$-projections of simulated nuclear positions. B, Tracing of a fixed preparation of a S. gregaria at 16 hours after egg laying (AEL) at $29^{\circ} \mathrm{C}$ and imaged in a manner that captured a subset of the $z$ depth of the embryo ${ }^{59}$. C, Selected time points of simulated blastoderm formation in an ellipsoid with the length and width of the D. melanogaster embryo. Left column: 3D renderings of pulling clouds, colored by instantaneous speed. Right column: $z$-projections of simulated nuclear positions. D, Tracings of published micrographs of D. melanogaster embryos from cycle 6, 7 , and 8 from Baker and colleagues ${ }^{21}$ (cycles 6 and 8 ) and Deneke and colleagues ${ }^{19}$ (cycle 7). In both sources, a subset of the $z$ depth of the embryo was imaged ${ }^{19,21}$. For each cycle, the total number of nuclei is shown in parentheses ${ }^{16}$. 


\section{References}

1. Reinsch, S. \& Gönczy, P. Mechanisms of nuclear positioning. Journal of Cell Science 111, 2283-2295 (1998).

2. Morris, N. R. Nuclear migration: from fungi to the mammalian brain. The Journal of Cell Biology 148, 1097$1102(2000)$.

3. Fischer, R. Nuclear movement in filamentous fungi. FEMS Microbiology Reviews 23, 39-68 (1999).

4. Roman, W. \& Gomes, E. R. Nuclear positioning in skeletal muscle. Seminars in Cell छ Developmental Biology 82, 51-56 (2018).

5. Bernard, F., Lepesant, J.-A. \& Guichet, A. Nucleus positioning within Drosophila egg chamber. Seminars in Cell छ Developmental Biology 82, 25-33 (2018).

6. Płachno, B. J. \& Świątek, P. Syncytia in plants: cell fusion in endosperm-placental syncytium formation in Utricularia (Lentibulariaceae). Protoplasma 248, 425-435 (2011).

7. Scholtz, G. \& Wolff, C. in Arthropod Biology and Evolution: Molecules, Development, Morphology 63-89 (Springer, 2013).

8. Kim, J. H., Jin, P., Duan, R. \& Chen, E. H. Mechanisms of myoblast fusion during muscle development. Current Opinion in Genetics $\sigma^{2}$ Development 32, 162-170 (2015).

9. Strom, N. B. \& Bushley, K. E. Two genomes are better than one: history, genetics, and biotechnological applications of fungal heterokaryons. Fungal Biology and Biotechnology 3, 4 (2016).

10. Anderson, D. The development of hemimetabolous insects. Developmental Systems: Insects 1, 95-163 (1972).

11. Anderson, D. The development of holometabolous insects. Developmental Systems: Insects 1, 165-242 (1972).

12. Johannsen, O. A. \& Butt, F. H. Embryology of Insects and Myriapods (McGraw-Hill Book Co., 1941).

13. Foe, V., Odell, G. \& Edgar, B. in The Development of Drosophila melanogaster 149-300 (Cold Spring Harbor Laboratory Press, Cold Spring Harbor, NY, 1993).

14. Lv, Z., de-Carvalho, J., Telley, I. A. \& Großhans, J. Cytoskeletal mechanics and dynamics in the Drosopbila syncytial embryo. Journal of Cell Science 134 (2021).

15. Sonnenblick, B. in Biology of Drosophila (ed Demerec, M.) 62-163 (John Wiley \& Sons, New York, NY, 1950).

16. Zalokar, M. Division and migration of nuclei during early embryogenesis of Drosophila melanogaster.J. Microsc. Biol. Cell. 25, 97-106 (1976).

17. Von Dassow, G. \& Schubiger, G. How an actin network might cause fountain streaming and nuclear migration in the syncytial Drosophila embryo. The Journal of Cell Biology 127, 1637-1653 (1994).

18. Royou, A., Sullivan, W. \& Karess, R. Cortical recruitment of nonmuscle myosin II in early syncytial Drosophila embryos: its role in nuclear axial expansion and its regulation by Cdc2 activity. The Journal of Cell Biology 158, 127-137 (2002).

19. Deneke, V. E. et al. Self-organized nuclear positioning synchronizes the cell cycle in Drosophila embryos. Cell 177, 925-941 (2019).

20. Telley, I. A., Gáspár, I., Ephrussi, A. \& Surrey, T. Aster migration determines the length scale of nuclear separation in the Drosophila syncytial embryo. The Journal of Cell Biology 197, 887-895 (2012).

21. Baker, J., Theurkauf, W. E. \& Schubiger, G. Dynamic changes in microtubule configuration correlate with nuclear migration in the preblastoderm Drosophila embryo. The Journal of Cell Biology 122, 113-121 (1993).

22. Foe, V. E. \& Alberts, B. M. Studies of nuclear and cytoplasmic behaviour during the five mitotic cycles that precede gastrulation in Drosophila embryogenesis. Journal of Cell Science 61, 31-70 (1983).

23. Foe, V. E., Field, C. M. \& Odell, G. M. Microtubules and mitotic cycle phase modulate spatiotemporal distributions of F-actin and myosin II in Drosophila syncytial blastoderm embryos. Development 127, 17671787 (2000).

24. Kanesaki, T., Edwards, C. M., Schwarz, U. S. \& Grosshans, J. Dynamic ordering of nuclei in syncytial embryos: a quantitative analysis of the role of cytoskeletal networks. Integrative Biology 3, 1112-1119 (2011).

25. Kaiser, F. et al. Mechanical model of nuclei ordering in Drosophila embryos reveals dilution of stochastic forces. Biophysical Journal 114, 1730-1740 (2018). 
26. Dutta, S., Djabrayan, N. J.-V., Torquato, S., Shvartsman, S. Y. \& Krajnc, M. Self-similar dynamics of nuclear packing in the early Drosophila embryo. Biophysical Journal 117, 743-750 (2019).

27. Lv, Z. et al. The emergent yo-yo movement of nuclei driven by cytoskeletal remodeling in pseudo-synchronous mitotic cycles. Current Biology 30, 2564-2573 (2020).

28. Deshpande, O., de-Carvalho, J., Vieira, D. V. \& Telley, I. A. Astral microtubule crosslinking by Feo safeguards uniform nuclear distribution in the Drosophila syncytium. bioRxiv (2020).

29. de-Carvalho, J., Tlili, S., Hufnagel, L., Saunders, T. E. \& Telley, I. A. Aster repulsion drives local ordering in an active system. bioRxiv (2020).

30. Kessel, E. L. The embryology of fleas. Smithsonian Miscellaneous Collections 98 (1939).

31. Eastham, L. A contribution to the embryology of Pieris rapae. Quarterly Journal of Microscopical Science 71, 353-394 (1927).

32. Wolf, R. Migration and division of cleavage nuclei in the gall midge, Wachtliella persicariae II. Origin and ultrastructure of the migration cytaster. Wilhelm Roux' Archiv für Entwicklungsmechanik der Organismen 188, 65-73 (1980).

33. Markow, T. A., Beall, S. \& Matzkin, L. M. Egg size, embryonic development time and ovoviviparity in Drosophila species. Journal of Evolutionary Biology 22, 430-434 (2009).

34. Donoughe, S. \& Extavour, C. G. Embryonic development of the cricket Gryllus bimaculatus. Developmental Biology 411, 140-156 (2016).

35. Nakamura, T. et al. Imaging of transgenic cricket embryos reveals cell movements consistent with a syncytial patterning mechanism. Current Biology 20, 1641-1647 (2010).

36. Wolff, C. et al. Multi-view light-sheet imaging and tracking with the MaMuT software reveals the cell lineage of a direct developing arthropod limb. eLife 7 (2018).

37. Berg, S. et al. ilastik: Interactive machine learning for (bio) image analysis. Nature Methods 16, 1226-1232 (2019).

38. Gönczy, P., Pichler, S., Kirkham, M. \& Hyman, A. A. Cytoplasmic dynein is required for distinct aspects of MTOC positioning, including centrosome separation, in the one cell stage Caenorbabditis elegans embryo. The Journal of Cell Biology 147, 135-150 (1999).

39. Kimura, K. \& Kimura, A. Intracellular organelles mediate cytoplasmic pulling force for centrosome centration in the Caenorhabditis elegans early embryo. Proceedings of the National Academy of Sciences of the USA 108, 137-142 (2011).

40. Gundersen, G. G. \& Worman, H. J. Nuclear positioning. Cell 152, 1376-1389 (2013).

41. De Simone, A., Spahr, A., Busso, C. \& Gönczy, P. Uncovering the balance of forces driving microtubule aster migration in C. elegans zygotes. Nature Communications 9, 938 (2018).

42. Potter, C. J., Tasic, B., Russler, E. V., Liang, L. \& Luo, L. The Q system: a repressible binary system for transgene expression, lineage tracing, and mosaic analysis. Cell 141, 536-548 (2010).

43. Donoughe, S., Kim, C. \& Extavour, C. G. High-throughput live-imaging of embryos in microwell arrays using a modular specimen mounting system. Biology Open 7, bio031260 (2018).

44. Preibisch, S., Saalfeld, S., Schindelin, J. \& Tomancak, P. Software for bead-based registration of selective plane illumination microscopy data. Nature Methods 7, 418-419 (2010).

45. Preibisch, S. et al. Efficient Bayesian-based multiview deconvolution. Nature Methods 11, 645-648 (2014).

46. Schindelin, J. et al. Fiji: an open-source platform for biological-image analysis. Nature Methods 9, 676-682 (2012).

47. Ji, J.-Y., Squirrell, J. M. \& Schubiger, G. Both cyclin B levels and DNA-replication checkpoint control the early embryonic mitoses in Drosophila. Development 131, 401-411 (2004).

48. Edgar, B. A., Odell, G. M. \& Schubiger, G. Cytoarchitecture and the patterning of fushi tarazu expression in the Drosophila blastoderm. Genes $\sigma^{\circ}$ Development 1, 1226-1237 (1987).

49. Sullivan, W., Fogarty, P. \& Theurkauf, W. Mutations affecting the cytoskeletal organization of syncytial Drosophila embryos. Development 118, 1245-1254 (1993). 
50. Riggs, B. et al. Actin cytoskeleton remodeling during early Drosophila furrow formation requires recycling endosomal components Nuclear-fallout and Rab11. The Journal of Cell Biology 163, 143-154 (2003).

51. Buttrick, G. J. et al. Akt regulates centrosome migration and spindle orientation in the early Drosophila melanogaster embryo. The Journal of Cell Biology 180, 537-548 (2008).

52. Anderson, C. A. et al. Nuclear repulsion enables division autonomy in a single cytoplasm. Current Biology 23, 1999-2010 (2013).

53. Koke, C., Kanesaki, T., Grosshans, J., Schwarz, U. S. \& Dunlop, C. M. A computational model of nuclear self-organisation in syncytial embryos. Journal of Theoretical Biology 359, 92-100 (2014).

54. Plamann, M., Minke, P. F., Tinsley, J. H. \& Bruno, K. S. Cytoplasmic dynein and actin-related protein Arp1 are required for normal nuclear distribution in filamentous fungi. The Journal of Cell Biology 127, 139-149 (1994).

55. Morris, N. R., Efimov, V. P. \& Xiang, X. Nuclear migration, nucleokinesis and lissencephaly. Trends in Cell Biology 8, 467-470 (1998).

56. Inoue, S., Turgeon, B., Yoder, O., et al. Role of fungal dynein in hyphal growth, microtubule organization, spindle pole body motility and nuclear migration. Journal of Cell Science 111, 1555-1566 (1998).

57. Burakov, A., Nadezhdina, E., Slepchenko, B. \& Rodionov, V. Centrosome positioning in interphase cells. The Journal of Cell Biology 162, 963-969 (2003).

58. Kimura, A. \& Onami, S. Computer simulations and image processing reveal length-dependent pulling force as the primary mechanism for C. elegans male pronuclear migration. Developmental Cell 8, 765-775 (2005).

59. Ho, K., Dunin-Borkowski, O. M. \& Akam, M. Cellularization in locust embryos occurs before blastoderm formation. Development 124, 2761-2768 (1997).

60. Roonwal, M. L. X-Studies on the embryology of the African migratory locust, Locusta migratoria migratorioides $\mathrm{R}$. and F. I-The early development, with a new theory of multi-phased gastrulation among insects. Philosophical Transactions of the Royal Society of London. Series B, Biological Sciences (1936).

61. Dawes, R., Dawson, I., Falciani, F., Tear, G. \& Akam, M. Dax, a locust Hox gene related to fushi-tarazu but showing no pair-rule expression. Development 120, 1561-1572 (1994).

62. Chodagam, S., Royou, A., Whitfield, W., Karess, R. \& Raff, J. The centrosomal protein CP190 regulates myosin function during early Drosophila development. Current Biology 15, 1308-1313 (2005).

63. Ji, J.-Y., Haghnia, M., Trusty, C., Goldstein, L. S. \& Schubiger, G. A genetic screen for suppressors and enhancers of the Drosophila cdk1-cyclin B identifies maternal factors that regulate microtubule and microfilament stability. Genetics 162, 1179-1195 (2002).

64. Edgar, B. A., Kiehle, C. P. \& Schubiger, G. Cell cycle control by the nucleo-cytoplasmic ratio in early Drosophila development. Cell 44, 365-372 (1986).

65. Huang, J.-y. \& Raff, J. W. The disappearance of cyclin B at the end of mitosis is regulated spatially in Drosophila cells. The EMBO Journal 18, 2184-2195 (1999).

66. Sarashina, I. et al. Location of micropyles and early embryonic development of the two-spotted cricket Gryllus bimaculatus (Insecta, Orthoptera). Development, Growth छ Differentiation 47, 99-108 (2005).

67. Ewen-Campen, B., Donoughe, S., Clarke, D. N. \& Extavour, C. G. Germ cell specification requires zygotic mechanisms rather than germ plasm in a basally branching insect. Current Biology 23, 835-842 (2013).

68. Pechmann, M. et al. Striking parallels between dorsoventral patterning in Drosophila and Gryllus reveal a complex evolutionary history behind a model gene regulatory network. eLife 10, e68287 (2021).

69. Church, S. H., Donoughe, S., de Medeiros, B. A. \& Extavour, C. G. Insect egg size and shape evolve with ecology but not developmental rate. Nature 571, 58 (2019).

70. Church, S. H., Donoughe, S., de Medeiros, B. A. \& Extavour, C. G. A dataset of egg size and shape from more than 6,700 insect species. Scientific Data 6, 1-11 (2019).

71. Cadot, B., Gache, V. \& Gomes, E. R. Moving and positioning the nucleus in skeletal muscle-one step at a time. Nucleus 6, 373-381 (2015). 
bioRxiv preprint doi: https://doi.org/10.1101/2021.04.26.441395; this version posted April 28, 2021. The copyright holder for this preprint (which

was not certified by peer review) is the author/funder, who has granted bioRxiv a license to display the preprint in perpetuity. It is made available under aCC-BY-NC-ND 4.0 International license.

Donoughe et al. 2021 - Cricket syncytial blastoderm formation

page 21 of 18

72. Bone, C. R. \& Starr, D. A. Nuclear migration events throughout development. Journal of Cell Science 129, 1951-1961 (2016).

73. Xiang, X. Nuclear movement in fungi. Seminars in Cell $\Xi^{2}$ Developmental Biology 82, 3-16 (2018).

74. Kokkoris, V., Stefani, F., Dalpé, Y., Dettman, J. \& Corradi, N. Nuclear dynamics in the arbuscular mycorrhizal fungi. Trends in Plant Science 25, 765-778 (2020).

75. Kokkoris, V. et al. Host identity influences nuclear dynamics in arbuscular mycorrhizal fungi. Current Biology 31, 1531-1538 (2021). 